\title{
Corrigendum: EpCAM-dependent extracellular vesicles from intestinal epithelial cells maintain intestinal tract immune balance
}

Lingling Jiang, Yingying Shen, Danfeng Guo, Diya Yang, Jiajun Liu, Xuefeng Fei, Yunshan Yang, Buyi Zhang, Zhendong Lin, Fei Yang, Xiaojian Wang, Keyi Wang, Jianli Wang \& Zhijian Cai

Nature Communications 7:13045 doi:10.1038/ncomms13045 (2016); Published 10 Oct 2016; Updated 14 Jun 2017

This Article contains an error in Fig. 8a, for which we apologize. In Fig. 8a, the control 3 image for p-ERK staining was inadvertently duplicated from the control 1 image of p-ERK staining. The correct version of this figure appears below as Fig. 1 .

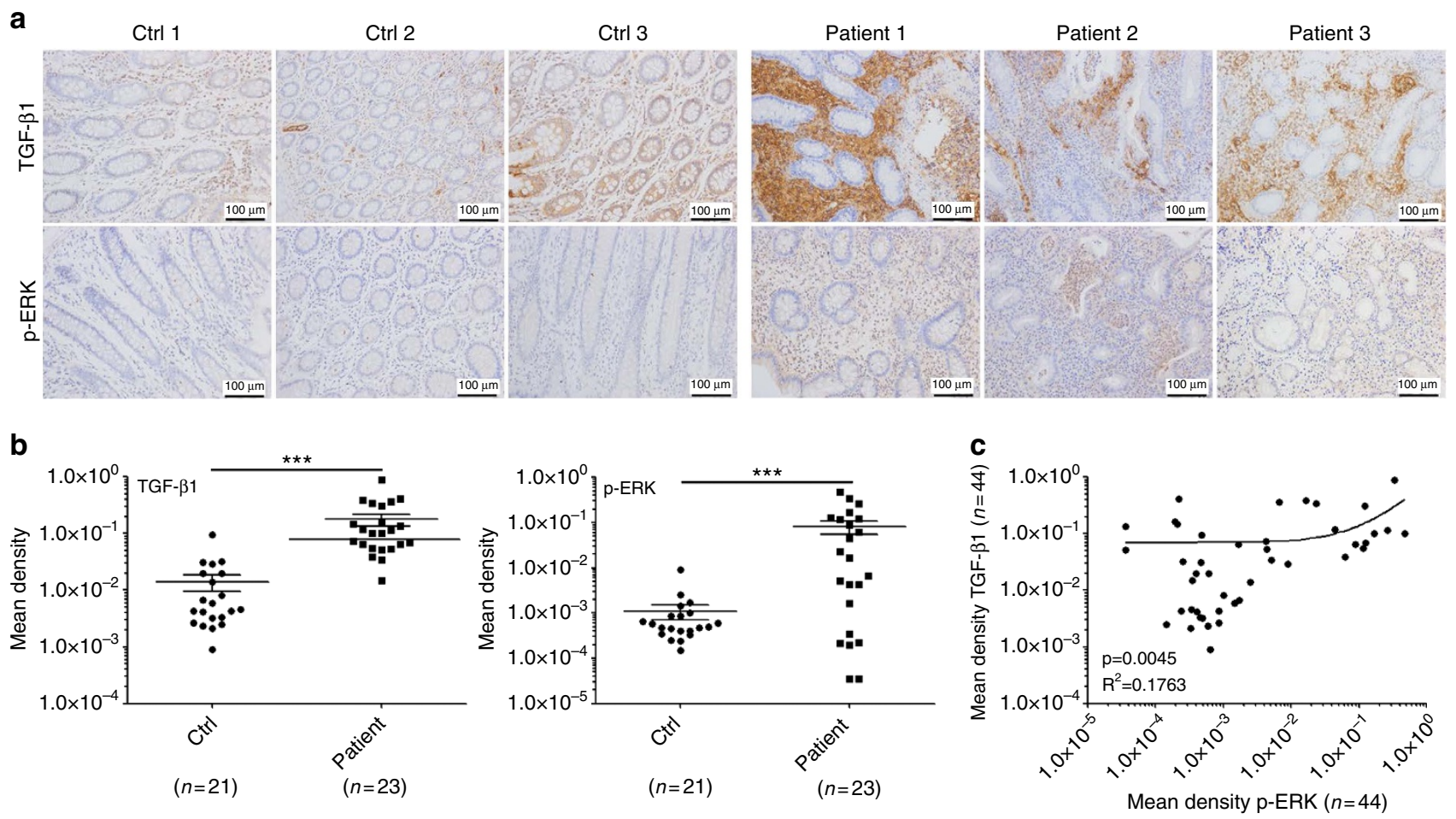

Figure 1 | 